\title{
Plasma Exchange to Rescue Patients with Autoantibodies Against Type I Interferons and Life-Threatening COVID-19 Pneumonia
}

\author{
Nicolas de Prost ${ }^{1}$ (D) Paul Bastard ${ }^{2,3,4} \cdot$ Romain Arrestier $^{1} \cdot$ Slim Fourati ${ }^{5,6} \cdot$ Mathieu Mahévas $^{7,8} \cdot$ Sonia Burrel $^{9}$. \\ Karim Dorgham ${ }^{10}$. Guy Gorochov ${ }^{10,11}$ - Yacine Tandjaoui-Lambiotte ${ }^{12} \cdot$ Iname Azzaoui ${ }^{8} \cdot$ Ignacio Fernandes $^{13}$. \\ Alain Combes ${ }^{14}$ • Jean-Laurent Casanova ${ }^{2,3,4,15,16}$. Armand Mekontso-Dessap ${ }^{1} \cdot$ Charles-Edouard Luyt $^{14}$
}

Received: 4 January 2021 / Accepted: 10 February 2021 / Published online: 22 February 2021

(C) The Author(s), under exclusive licence to Springer Science+Business Media, LLC part of Springer Nature 2021

\begin{abstract}
Purpose To report four cases of life-threatening COVID-19 pneumonia in patients with high blood concentrations of neutralizing autoantibodies against type I interferons (IFNs), who were treated with plasma exchange (PE) as a rescue therapy.

Methods Prospective case series, which included patients, diagnosed with RT-PCR-confirmed SARS-CoV-2 infection and positive autoantibodies against type I IFNs in two French intensive care units (ICUs) between October 8 and November 14, 2020. Six critically ill COVID-19 patients with no anti-IFN antibodies were used as controls. Anti-IFN autoantibodies and IFN concentrations, together with the levels of anti-SARS-CoV-2 antibodies, were measured sequentially in serum. Viral load was determined in the upper and lower respiratory tract. Patients were followed during hospital stay.

Results Three men and one woman were included. Three of the patients had four PE sessions each, while another had three PE sessions. PE decreased the concentrations of autoantibodies against type I IFN in all four patients, whereas anti-SARS-CoV-2 antibody levels remained stable. Autoantibodies against type I IFN levels were high in tracheal aspirates of one patient and decreased after three PE sessions. By contrast, anti-IFN autoantibodies were not detected in tracheal aspirates from five control patients without detectable anti-IFN autoantibodies in serum. During PE, serum IFN- $\alpha$ levels slightly increased in three out of
\end{abstract}

Nicolas de Prost

nicolas.de-prost@aphp.fr

1 From the Service de Médecine Intensive Réanimation, Hôpitaux Universitaires Henri Mondor, Assistance Publique - Hôpitaux de Paris (AP-HP) and Groupe de Recherche Clinique CARMAS, Faculté de Santé de Créteil,, Université Paris Est Créteil, 51, Avenue du Maréchal de Lattre de Tassigny, 94010 Créteil Cedex, France

2 Laboratory of Human Genetics of Infectious Diseases, Necker Branch, INSERM U1163, Necker Hospital for Sick Children, Paris, France

3 Imagine Institute, University of Paris, Paris, France

4 St. Giles Laboratory of Human Genetics of Infectious Diseases, Rockefeller Branch, The Rockefeller University, New York, NY, USA

5 Service de Virologie, Département de prévention, diagnostic et traitement des infections, Hôpitaux Universitaires Henri Mondor, Assistance Publique - Hôpitaux de Paris (AP-HP), Créteil, France

6 INSERM U955 (team 18) and Faculté de Santé de Créteil, Université Paris Est Créteil, 94010 Créteil Cedex, France

7 Service de Médecine Interne, Hôpitaux Universitaires Henri Mondor, Assistance Publique - Hôpitaux de Paris (AP-HP), Faculté de Santé de Créteil, Université Paris Est Créteil, 94010 Créteil Cedex, France
8 INSERM U955 and Faculté de Santé de Créteil, Université Paris Est Créteil, 94010 Créteil Cedex, France

9 Assistance Publique-Hôpitaux de Paris, Hôpital Pitié-Salpêtrière, Département de Virologie, Sorbonne Université, INSERM U1136, Institut Pierre Louis d'Epidémiologie et de Santé Publique (IPLESP), Paris, France

10 Sorbonne Université, Inserm, Centre d'Immunologie et des Maladies Infectieuses (CIMI-Paris), 75013 Paris, France

11 Département d'Immunologie, Assistance Publique Hôpitaux de Paris (AP-HP), Hôpital Pitié-Salpêtrière, 75013 Paris, France

12 Réanimation médico-chirurgicale, Assistance Publique-Hôpitaux de Paris, Hôpital Avicenne and INSERM U1272 Hypoxia \& Lung, Bobigny, France

13 Structural Virology Unit, Institut Pasteur, and CNRS UMR 3569, Paris, France

14 Sorbonne Université, Assistance Publique-Hôpitaux de Paris, Hôpital Pitié-Salpêtrière, Médecine Intensive Réanimation, AP-HP and INSERM UMRS_1166-iCAN, Institute of Cardiometabolism and Nutrition, Paris, France

15 Pediatric Hematology and Immunology Unit, Necker Hospital for Sick Children, AP-HP, Paris, France

16 Howard Hughes Medical Institute, New York, NY, USA 
four patients, and upper respiratory tract viral load decreased in all patients. All patients were alive at day 28 of ICU admission. Two patients eventually died in the ICU, while the two survivors were discharged from the ICU at days 50 and 66.

Conclusions PE efficiently removes autoantibodies against type I IFNs, including those detected in tracheal aspirates, without affecting anti-SARS-CoV-2 antibody levels, in patients with life-threatening COVID-19 pneumonia. The clinical benefit of PE in patients with autoantibodies against type I IFNs should be tested in a larger study.

Keywords SARS-CoV-2 $\cdot$ COVID-19 $\cdot$ type I interferons $\cdot$ plasma exchanges

Autoantibodies (auto-Abs) against type I interferons (IFN) have recently been shown to underlie life-threatening coronavirus disease 2019 (COVID-19) pneumonia in at least $10 \%$ of patients [1]. Inborn errors of TLR3- and IRF7-dependent type I IFN immunity also underlie life-threatening COVID-19, in a smaller proportion of patients [2]. Auto-Abs were detected in $12.5 \%$ of men and $2.6 \%$ of women with life-threatening disease, but in none of 663 patients with asymptomatic or mild SARS-CoV-2 (severe acute respiratory coronavirus 2) infection, and in only $0.3 \%$ of healthy individuals aged 20 to 65 years [1]. These antibodies neutralized type I IFNs, including the ability of IFN- $\alpha 2$ to block SARS-CoV-2 infection, both in vitro and in vivo, with all 13 individual subtypes of IFN- $\alpha$ present at only very low levels or undetectable in the blood of these patients [1]. Collectively, these findings suggest that type I IFNs are essential for the control of SARS-CoV-2 infection. They also suggest a two-step model for the pathogenesis of life-threatening COVID-19 pneumonia, with defective type I IFN immunity in the first few days of infection underlying viral dissemination, leading to the unleashing of excessive pulmonary and systemic inflammation [3]. However, despite encouraging results in case reports of early therapy with subcutaneous Peg-IFN- $\alpha 2 \mathrm{a}$ in patients with inborn errors affecting the production of type I IFNs [4], the SOLIDARITY trial reported no beneficial effect of subcutaneous IFN- $\beta$ in a more general population of hospitalized patients with COVID-19 pneumonia, suggesting that an earlier administration might be beneficial [5].

As an alternative to treatment with exogenous type I IFNs, therapeutic interventions including monoclonal antibodies depleting plasmablasts, and the specific inhibition of type I IFNreactive $\mathrm{B}$ cells might be beneficial in patients with auto-Abs directed against type I IFN and SARS-CoV-2 infection. Here, we hypothesized that removing the auto-Abs directed against type I IFN in patients with SARS-CoV-2 infection might be of benefit to patients, by restoring endogenous type I IFN levels. Potential benefits of plasma exchange (PE) in patients with critical COVID-19 have been reported [6-9], but this treatment has never been studied in patients with auto-Abs against type I IFNs. PE is probably the fastest way to remove circulating auto-Abs against type I IFNs, but it also exposes the patient to potential adverse events [10], including a risk of decreasing the concentration of neutralizing Abs against SARS-CoV-2. Its safety and efficiency for removing autoAbs against type I IFNs in patients hospitalized in intensive care units (ICUs) for life-threatening COVID-19, and its impact on Abs against SARS-CoV-2 have not been assessed. It also remains unknown whether the depletion of auto-Abs against type I IFN would lead to an increase in blood type I IFN concentration in vivo and improve the outcome of patients with auto-Abs against type I IFN and life-threatening COVID-19 pneumonia, given that inflammation is probably of greater concern than viral replication at this late stage of the disease [11]. We report here four cases of life-threatening COVID-19 pneumonia in patients with high concentrations of neutralizing auto-Abs against type I IFNs who were treated with $\mathrm{PE}$ as a rescue therapy.

\section{Methods}

Supplemental methods are available in the online supplement.

\section{Patients and Controls}

The four patients were recruited from two French ICUs between October 8 and November 14, 2020. All had lifethreatening COVID-19 pneumonia with the acute respiratory distress syndrome (ARDS) and a rapid worsening of clinical condition together with high serum anti-IFN- $\alpha$ autoantibody concentrations and persisting SARS-CoV-2 replication. All had received or were on a 10-day course of $6 \mathrm{mg}$ /day dexamethasone [12]. No other immune modulating or antiviral therapy was administered. For each patient, the decision to perform PE was made by the clinicians in charge of patient care.

Six critically ill COVID-19 patients with ARDS and no anti-IFN antibodies measured on one serum sample were used as controls. Their presentation at ICU admission and clinical course is detailed in supplemental Table 2 . They received the same course of dexamethasone than the four patients. Five additional controls were used for measuring tracheal concentrations of anti-IFN autoantibodies. 


\section{Plasma Exchange}

PE was performed by continuous flow centrifugation for three patients and by plasma filtration for the fourth (supplementary Table 1). Anti-IFN autoantibodies and IFN concentrations were measured in serum before, $1 \mathrm{~h}$ and $24 \mathrm{~h}$ after each PE session, as well as in the PE effluent, and in the lower respiratory tract of one patient at various time points. Viral load was determined in blood samples from the patients, and in the upper (nasopharyngeal sampling) and lower (bronchoalveolar lavage (BAL) fluid) respiratory tract before, during, and after PE. The levels of anti-SARS-CoV-2 antibodies (anti-nucleocapsid $(\mathrm{N})$ and anti-spike (S) antibodies) were determined before and after each PE session and later on during the stay of the patients in the ICU. The primary clinical endpoint was vital status at ICU day 28. Patients were followed throughout their hospital stay.

\section{SARS-CoV-2 Viral Load}

SARS-CoV-2 viral load was determined in the upper respiratory tract by nasopharyngeal sampling with swabs, and in the lower respiratory tract by BAL, as previously described [13]. Details are provided in the online supplement. The cycle threshold (CT) values for RT-PCR were used as indicators of the viral load of SARS-CoV-2 RNA, with lower cycle threshold values corresponding to higher viral loads.

\section{Anti-IFN Auto-antibodies}

ELISA was performed as previously described [1]. Details are provided in the online supplement.

\section{Blood IFN-a Concentration}

Serum IFN- $\alpha$ concentrations were determined by digital ELISA with Simoa technology, as previously described [14, 15], with reagents and procedures obtained from the Quanterix Corporation ${ }^{\circledR}$.

\section{Anti-SARS-CoV-2 Antibody Assays}

Serum samples were analyzed for the detection of IgG antibodies against the SARS-CoV-2 $\mathrm{N}$ protein (ARCHITECT®, Abbott Laboratories) and for IgG antibodies against the SARS-CoV-2 spike (S) protein (VITROS®, Ortho Clinical Diagnostics). Details are provided in the online supplement.

\section{B Cell Response}

The B cell response to the SARS-CoV-2 spike protein was determined by analyses on blood before PE. Six critically ill
COVID-19 patients with ARDS and no anti-IFN antibodies were used as controls (supplemental Table 2).

\section{Ethical Considerations}

All subjects were recruited according to protocols approved by the competent local institutional review boards. All protocols conformed to local ethics recommendations and informed consent was obtained when required.

\section{Results}

The baseline clinical characteristics of the patients are displayed in Table 1 and their clinical course is depicted in Fig. 1. Three men and one woman were included. All had life-threatening COVID-19 pneumonia with no improvement or with a decline in clinical state in the days preceding PE. Auto-Abs against type I IFNs were detected in serum samples from all four patients (Table 1). Three of the patients had four PE sessions each, with an interval of 24 to $48 \mathrm{~h}$ between sessions, whereas the fourth patient had three sessions. PE was initiated within 8 to 22 days of ICU admission.

Patient 1 (Fig. 2a) displayed a marked linear decrease in anti-IFN auto-Abs with PE, followed by a rebound after each session. Serum interferon- $\alpha$ concentration remained close to zero and did not vary over time. The serum concentrations of anti-SARS-CoV-2 antibodies (anti-S and anti-N) were unaffected by $\mathrm{PE}$ and remained stable over time. SARS-CoV-2 excretion decreased with time, becoming undetectable by day 26 of the patient's stay in the ICU. The respiratory status of this patient improved and he was eventually discharged alive from the ICU on day 45 (Fig. 1a).

Patient 2 (Fig. 2b) displayed a dramatic decrease in antiIFN auto-Abs titer after the first PE session. Auto-Ab levels subsequently leveled out to a plateau, with almost complete clearance observed after the fourth session. Serum interferon- $\alpha$ concentration, which was measurable only before and after the third and fourth PE sessions, was initially close to zero, but had increased by the end of the fourth session, when the titer of anti-IFN auto-Abs was lowest. Strikingly, the serum concentrations of anti-SARS-CoV-2 antibodies increased over time, highlighting that PE cleared antiIFN auto-Abs much more strongly than anti-N and anti-S antibodies. Viral load was initially high in the upper (PCR $\mathrm{CT}=15)$ and lower $(\mathrm{PCR} C \mathrm{C}=26)$ respiratory tract. This patient initially had viremia, but the virus was no longer detectable in the blood by day 23 in the ICU. However, the clinical status of this patient worsened after the second PE, with the development of refractory ARDS requiring endotracheal intubation and ECMO implantation. This patient was still alive at 
Table 1 Characteristics and outcomes of patients

\begin{tabular}{|c|c|c|c|c|}
\hline & Patient 1 & Patient 2 & Patient 3 & Patient 4 \\
\hline Age, years & 73 & 67 & 28 & 36 \\
\hline Sex & Male & Male & Female & Male \\
\hline Body mass index $\left(\mathrm{kg} / \mathrm{m}^{2}\right)$ & 34.0 & 24.4 & 25.6 & 31.0 \\
\hline Comorbidities and associated conditions & Obesity & Hypertension, diabetes & 28-week pregnancy & Obesity \\
\hline \multicolumn{5}{|l|}{ Characteristics at ICU admission } \\
\hline SAPS II & 36 & 38 & 29 & 51 \\
\hline SOFA score & 7 & 7 & 7 & 8 \\
\hline $\begin{array}{l}\text { Time from first symptoms to ICU admission, } \\
\text { days }\end{array}$ & 6 & 3 & 7 & 5 \\
\hline WHO 10-point CPS* $(0-10)$ & 9 & 5 & 8 & 8 \\
\hline Endotracheal intubation & Yes & No & Yes & Yes \\
\hline $\mathrm{PaO}_{2} / \mathrm{FiO}_{2}$ ratio, $\mathrm{mmHg}$ & 97 & - & 70 & 44 \\
\hline Shock & Yes & No & No & No \\
\hline Pulmonary co-infection & No & Streptococcus pneumoniae & No & No \\
\hline \multicolumn{5}{|l|}{ Biological data at ICU admission } \\
\hline White blood cell counts, G/L (4.0-10.0) & 7.1 & 11.2 & 22.4 & 16.2 \\
\hline Neutrophils, G/1 (1.5-7.0) & 5.7 & 10.8 & 19.0 & 13.8 \\
\hline Lymphocytes, G/L (1.0-4.0) & 0.8 & 0.3 & 1.4 & 1.9 \\
\hline Serum D-dimer level, ng/mL $(<500)$ & 1242 & 787 & $>10,000$ & 6447 \\
\hline Serum creatinine level, $\mu \mathrm{mol} / \mathrm{L}(<106)$ & 56 & 341 & 21 & 105 \\
\hline Serum urea level, $\mathrm{mmol} / \mathrm{L}(<11.9)$ & 6.8 & 23.9 & 4.0 & 7.1 \\
\hline \multicolumn{5}{|l|}{ Characteristics at first plasma exchange session } \\
\hline $\begin{array}{l}\text { SARS-CoV-2 RNA detection in the } \\
\text { respiratory tract, } \mathrm{Ct}\end{array}$ & 31.0 & 15.0 & 28 & 32.8 \\
\hline SARS-CoV-2 viremia & Negative & Positive & Positive & Negative \\
\hline Serum anti-interferon- $\alpha 2$ antibody, o.d. & 1.5 & 3.3 & 4.0 & 4.0 \\
\hline $\begin{array}{l}\text { Time from ICU admission to first session, } \\
\text { days }\end{array}$ & 22 & 12 & 11 & 8 \\
\hline WHO 10-point CPS* $(0-10)$ & 8 & 6 & 9 & 9 \\
\hline Ventilation mode & Invasive & Non-invasive & Invasive & Invasive \\
\hline $\mathrm{PaO}_{2} / \mathrm{FiO}_{2}$ ratio, $\mathrm{mmHg}$ & 113 & 74 & 88 & 80 \\
\hline SOFA score & 3 & 3 & 4 & 10 \\
\hline ECMO & No & No & Yes & Yes \\
\hline \multicolumn{5}{|l|}{ During or after plasma exchange } \\
\hline Number of plasma exchange sessions & 4 & 4 & 4 & 3 \\
\hline Adverse events attributed to plasma exchange & No & No & No & No \\
\hline Need for endotracheal intubation & NA & Yes & NA & NA \\
\hline Need for ECMO & No & Yes & NA & NA \\
\hline \multicolumn{5}{|l|}{ Outcomes } \\
\hline Duration of invasive mechanical ventilation & 44 & 49 & 49 & 15 \\
\hline Number of VAP episodes & 2 & 3 & 2 & 1 \\
\hline Putative invasive aspergillosis & No & Yes & No & No \\
\hline ECMO support & No & Yes & Yes & Yes \\
\hline Time between intubation and ECMO, days & - & 0 & 7 & 3 \\
\hline Duration of ECMO support, days & - & 49 & 42 & 7 \\
\hline Renal replacement therapy & No & Yes & No & No \\
\hline Vital status at ICU day 28 & Alive & Alive & Alive & Alive \\
\hline Vital status during ICU stay & $\begin{array}{l}\text { Survived (discharged at } \\
\text { day 45) }\end{array}$ & $\begin{array}{l}\text { Died (day } 65 \text { of ICU } \\
\quad \text { admission) }\end{array}$ & $\begin{array}{l}\text { Died (day } 49 \text { of ICU } \\
\text { admission) }\end{array}$ & $\begin{array}{l}\text { Survived (discharged at } \\
\text { day 14) }\end{array}$ \\
\hline
\end{tabular}

$C t$, cycle threshold value of the RT-PCR; SAPS, severe acute physiology score; SOFA, sequential organ failure assessment; ICU, intensive care unit; $E C M O$, extracorporeal membrane oxygenation; o.d., optical density; $V A P$, ventilator-associated pneumonia; $N A$, not applicable; SARS-CoV-2, severe acute respiratory syndrome coronavirus 2 ; *World Health Organization ten-category ordinal scale: 0 , uninfected; 1 , asymptomatic; viral RNA detected; 2, symptomatic; independent; 3 , not hospitalized with resumption of normal activities; 2 , not hospitalized, but unable to resume normal activities; 3 , symptomatic; assistance needed; 4, hospitalized, not requiring oxygen; 5 , hospitalized, requiring oxygen by mask or nasal prongs; 6 , hospitalized, requiring nasal high-flow oxygen therapy, noninvasive mechanical ventilation, or both; 7, hospitalized, requiring intubation and mechanical ventilation, $\mathrm{PaO}_{2} / \mathrm{FiO}_{2}>150 \mathrm{mmHg} \mathrm{OR} \mathrm{SpO} 2 / \mathrm{FiO}_{2} \geq 200 ; 8$, hospitalized, requiring mechanical ventilation, $\left(\mathrm{PaO}_{2} / \mathrm{FiO}_{2} \leq 150 \mathrm{mmHg} \mathrm{OR} \mathrm{SpO} 2 / \mathrm{FiO} 2<200\right) \mathrm{OR}$ vasopressors (norepinephrine less than $0.3 \mu \mathrm{g} / \mathrm{kg} / \mathrm{min}$ ); 9, mechanical ventilation, $\mathrm{PaO}_{2} / \mathrm{FiO}_{2}<150 \mathrm{mmHg}$ AND vasopressors (norepinephrine more than $0.3 \mu \mathrm{g} / \mathrm{kg} / \mathrm{min}$ ), OR Dialysis OR ECMO requiring; 10, dead

ICU day 28 but on mechanical ventilation and ECMO support. He eventually died at ICU day 65 of ICU admission of a non-documented septic shock (Fig. 1b).
Patient 3 (Fig. 2c) displayed a marked decrease in anti-IFN auto-Ab levels following PE, with an almost complete clearance of serum antibodies after the fourth session. Serum 

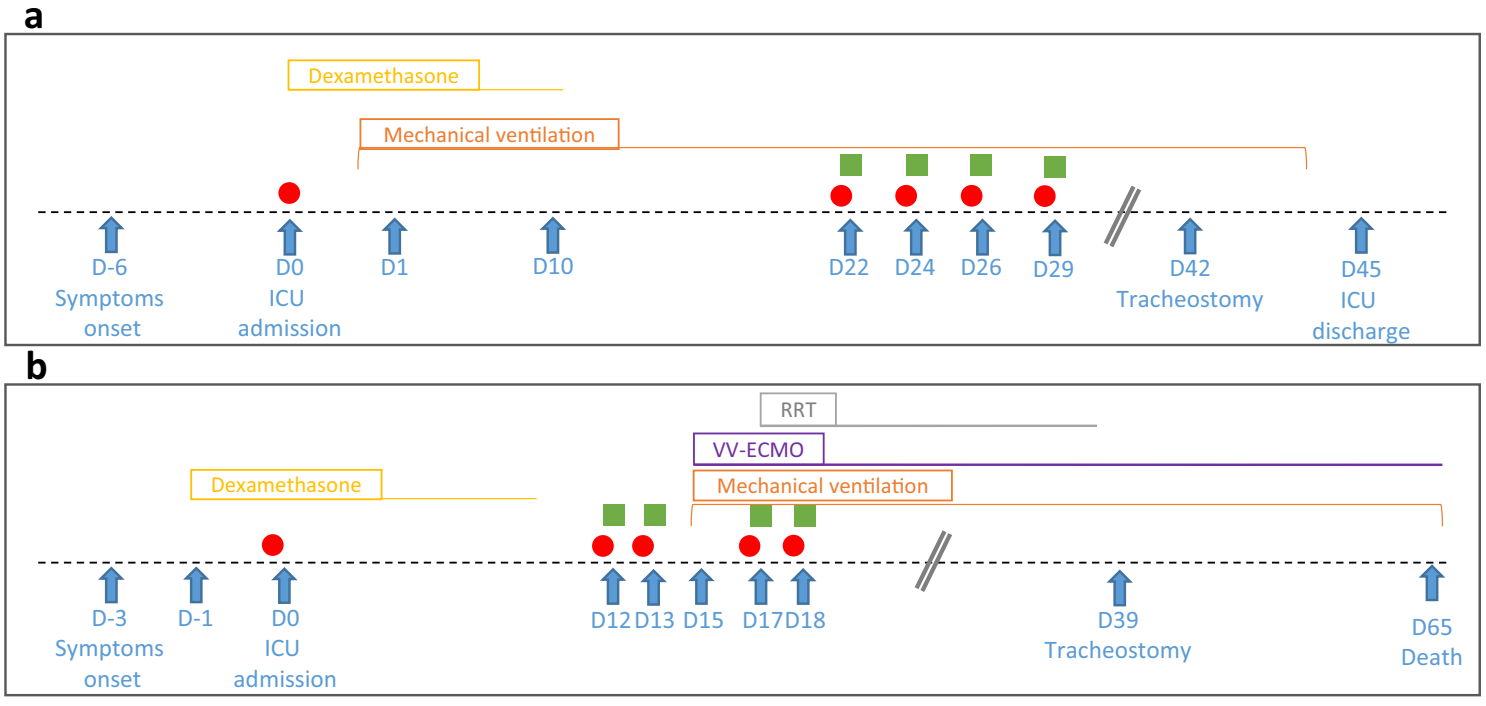

C

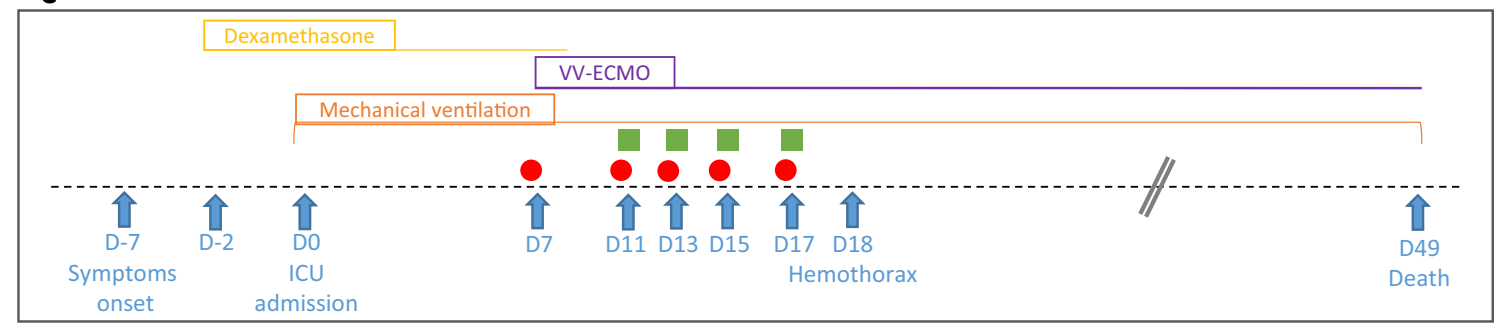

d

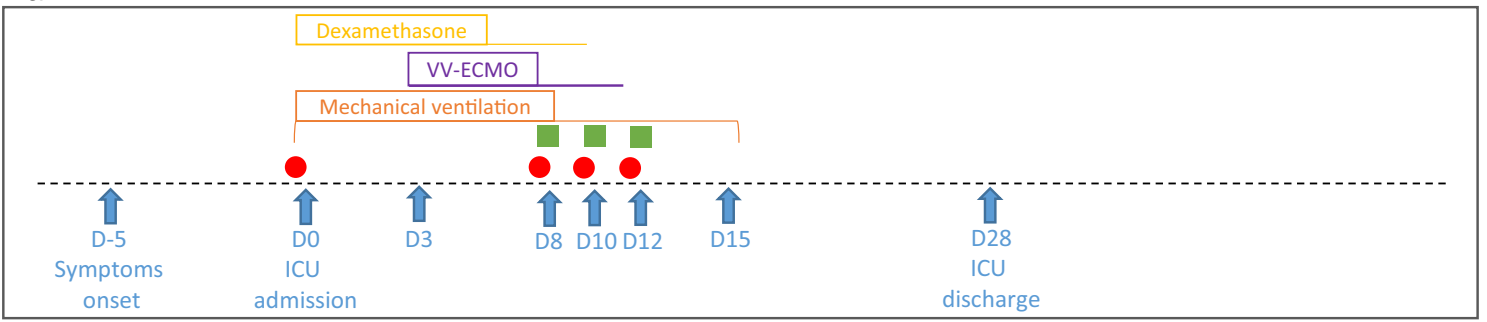

Type I Anti-IFN antibody sampling

Plasma exchange

Fig. 1 Clinical course of four critically ill patients with COVID-19 acute respiratory distress syndrome. Patients 1 (a), 2 (b), 3 (c), and 4 (d). D, day; RRT, renal replacement therapy; VV-ECMO, veno-venous-extra-corporeal membrane oxygenation

interferon- $\alpha$ concentration increased after the first PE session, when anti-IFN auto-Ab clearance was maximal and the virus was still being excreted, but remained close to zero thereafter. The serum concentration of anti-SARS-CoV-2 antibodies decreased moderately over time, and SARS-CoV-2 RT-PCR results became negative between days 15 and 18 in the ICU. The course of the disease was complicated and severe in this 28-year-old patient after PE. She remained on invasive mechanical ventilation and was dependent of veno-venous ECMO support on day 30 in the ICU. She developed a spontaneous hemothorax $24 \mathrm{~h}$ after the fourth PE, despite the use of $100 \%$ plasma for substitution (supplemental Table 1) and the absence of hemostasis disorders (plasma fibrinogen concentration was $3.7 \mathrm{~g} / \mathrm{L}$, prothrombin time $>80 \%$, platelet counts were $194 \mathrm{G} / \mathrm{L}$ ) to the exception that she was receiving heparin intravenously $(26,000 \mathrm{IU} / 24 \mathrm{~h}$ for an activated partial prothrombin time of 2.77). She was still alive at ICU day 28 . Her respiratory status did not improve and she remained dependent of ECMO support and finally died at ICU day 49 of a non-documented septic shock (Fig. 1c).

Patient 4 (Fig. 2d) also displayed a marked decrease in antiIFN auto-Ab levels after PE. Anti-IFN antibody levels were also measured in tracheal aspirates. They were high, but decreased progressively after three PE sessions. By contrast, anti-IFN auto-Abs were not detected in tracheal aspirates from five other patients hospitalized in the same ICU but without detectable auto-Abs against type I IFNs in plasma or serum (Fig. 3). Serum interferon- $\alpha$ concentrations were measurable 
a
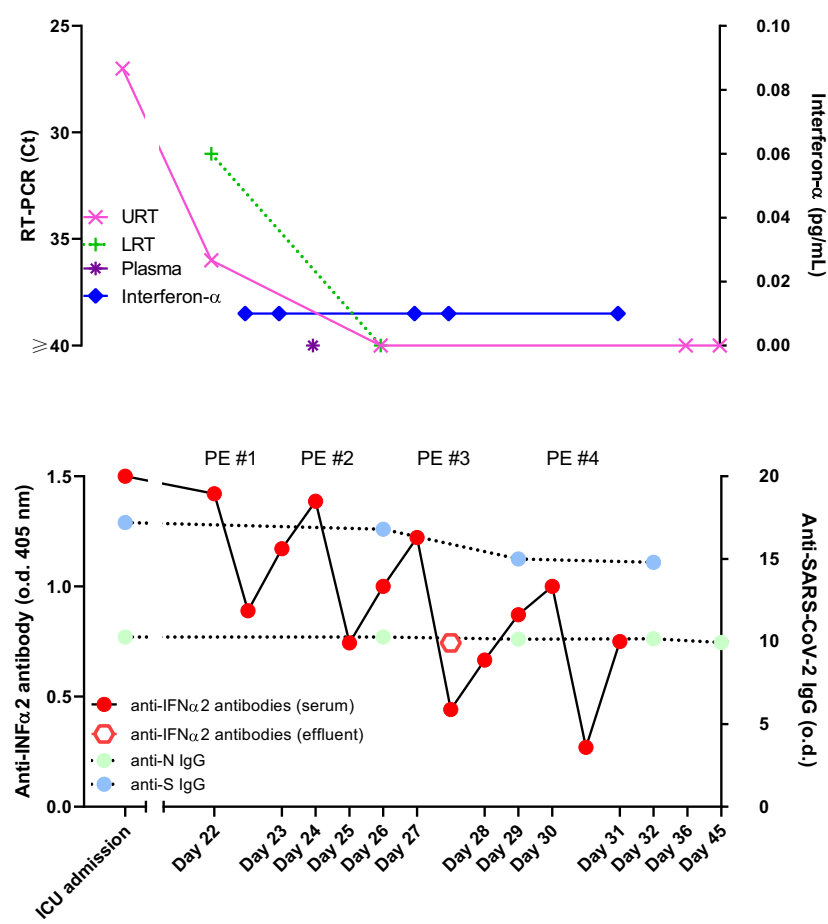

C
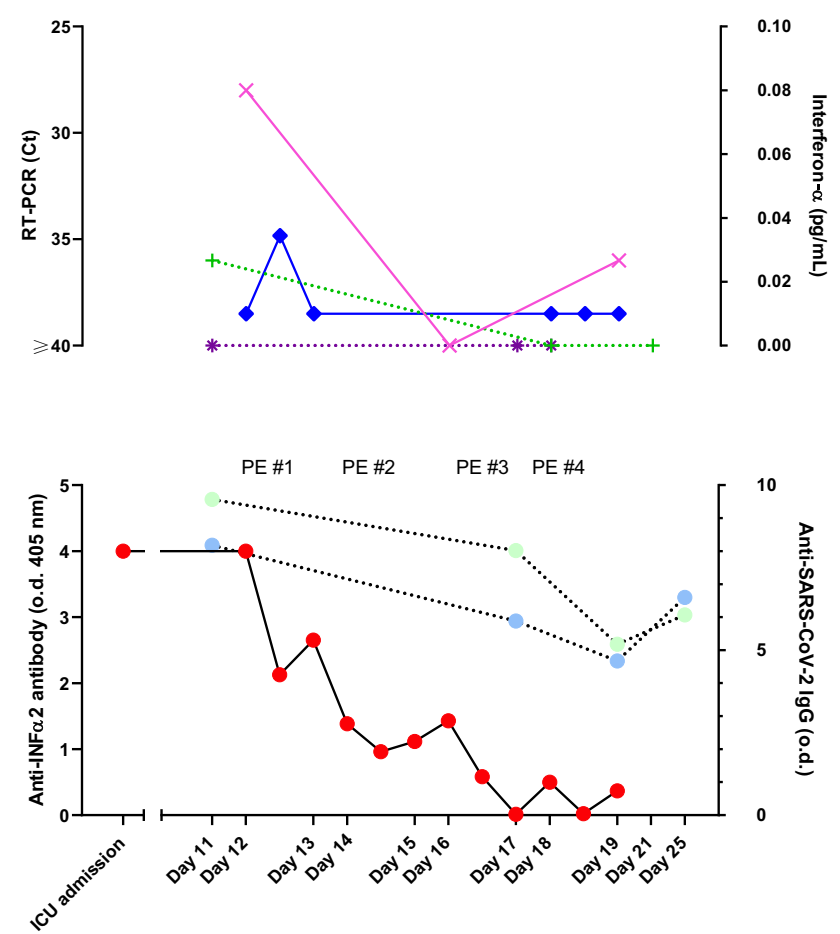

only before and after the third PE session, and a slight increase was observed. Serum concentration of anti-SARS-CoV-2 antibodies increased, and RT-PCR CT decreased over time. This patient was weaned off ECMO and mechanical ventilation 3 and 7 days, respectively, after the initiation of PE (Fig. 1d). b
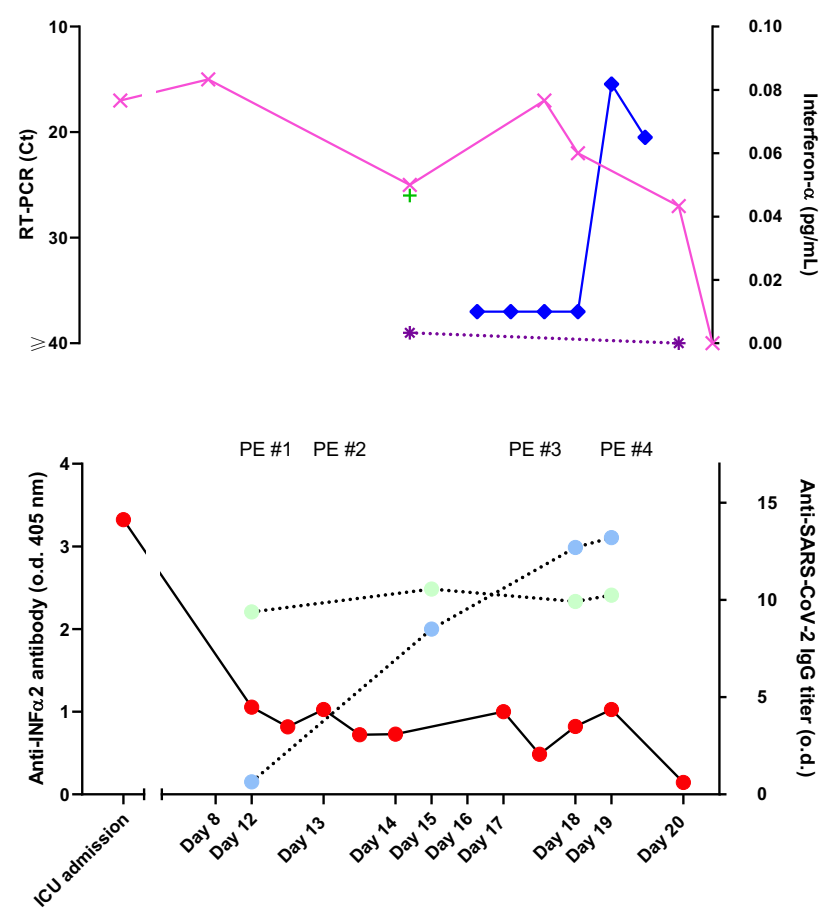

d
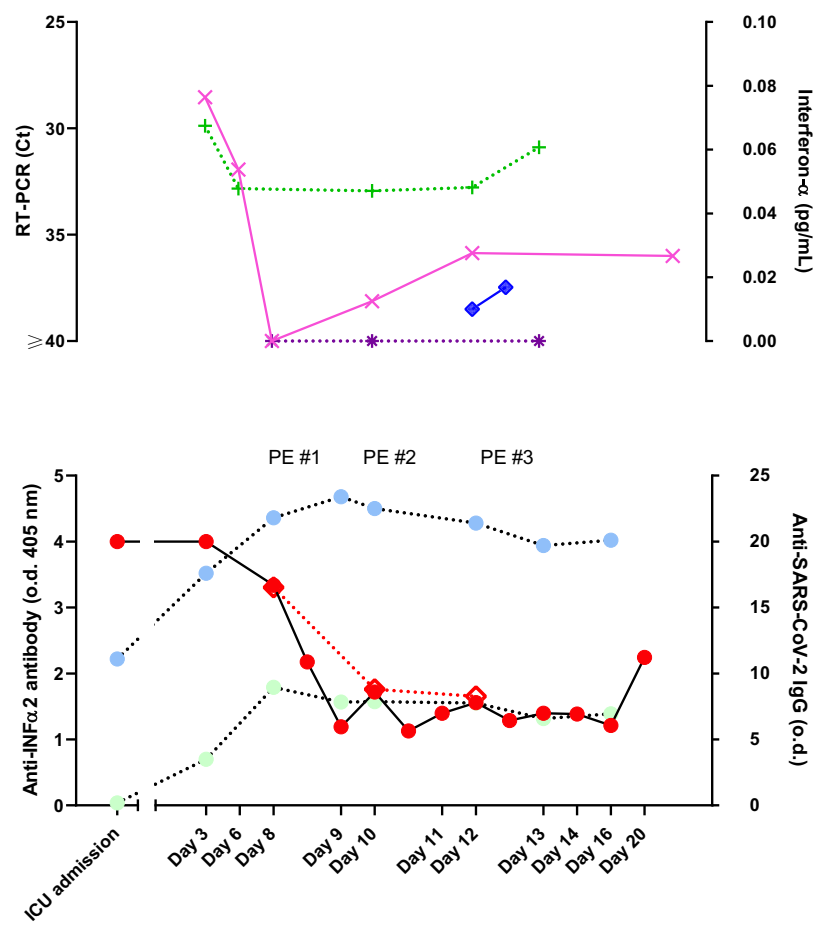

The sustained humoral anti-SARS-CoV-2 response observed over time, illustrated by the stability of or increase in serum concentrations of anti-N and anti-S antibodies (Fig. 1), was consistent with the normal response of $\mathrm{B}$ cells obtained from the four patients with anti-IFN auto-Abs to the SARS- 
Fig. 2 Time course of type I anti-interferon (IFN) and anti-SARS-CoV-2 antibodies, viral load in the upper and lower respiratory tract and serum interferon- $\alpha$ concentration in four critically ill patients with COVID-19 acute respiratory distress syndrome. Patients 1 (a), 2 (b), 3 (c), and 4 (d) displayed a marked decrease in serum concentrations of anti-IFN antibodies (lower panels, red circles connected by a continuous black line) as the plasma exchange sessions progressed. Over the same time period, the concentrations of anti-SARS-CoV-2 anti-nucleocapsid (N, green circles) and anti-spike ( $\mathrm{S}$, blue circles) antibodies remained stable (patient 1, a) or even increased (patients 2 and $4, \mathbf{b}, \mathbf{d}$ ), in three of the patients (the exception being patient 3 ) (c). Serum interferon- $\alpha$ concentration (upper panels, blue diamonds connected by a continuous blue line) did not vary significantly over time in patient 1 , but increased strongly in patient 2 , with only a transient increase observed in patient 3 .Viral excretion decreased over time (upper panels) in both the upper (pink symbols) and lower (green symbols) respiratory tract (URT, LRT). Note that the $y$-axis for the RT-PCR has been inverted to reflect the inverse correlation of cycle threshold (Ct) with RNA viral load. A Ct value $\geq 40$ was considered negative

Cov2 Spike protein, relative to six critically ill COVID-19 patients without anti-IFN antibodies (Fig. 4, supplemental Table 2).

\section{Discussion}

We report here the first experience of $\mathrm{PE}$ in patients with autoAbs against type I IFNs hospitalized in ICUs for lifethreatening COVID-19 pneumonia. PE efficiently decreased the concentrations of auto-Abs against type I IFNs in the blood of all these patients, but also, importantly, in tracheal aspirates in the patient tested. Auto-Abs against type I IFNs have never before been detected in tracheal aspirates, and this finding further suggests that these antibodies contributed to life-threatening COVID-19 pneumonia. By contrast, antiSARS-CoV-2 Abs, and neutralizing anti-S Abs in particular, seemed to be less affected by PE. Furthermore, these four patients with auto-Abs against type I IFNs had normal antiSARS-CoV-2 humoral responses, as shown by comparison to critically ill COVID-19 patients without anti-IFN auto-Abs. The observation that the depletion of anti-IFN antibodies was not associated with a significant decrease in serum antiSARS-CoV-2 antibody titers is important, because serum concentrations of anti-SARS-CoV-2 IgG have been shown to be associated with survival in critically ill COVID-19 patients [16]. Importantly, IFN- $\alpha$ became detectable in the serum samples of three patients following the depletion of anti-IFN autoAbs, suggesting that plasma exchange may restore endogenous type I IFN production and activity in vivo in patients with auto-Abs against type I IFNs. Nevertheless, the IFN- $\alpha$ levels remained low, which may reflect the persistence of autoantibodies and/or the late stage of disease.

These results are encouraging, but further studies are required to evaluate the safety of PE in critically ill COVID-19
COVID-19 ARDS

Patient 4

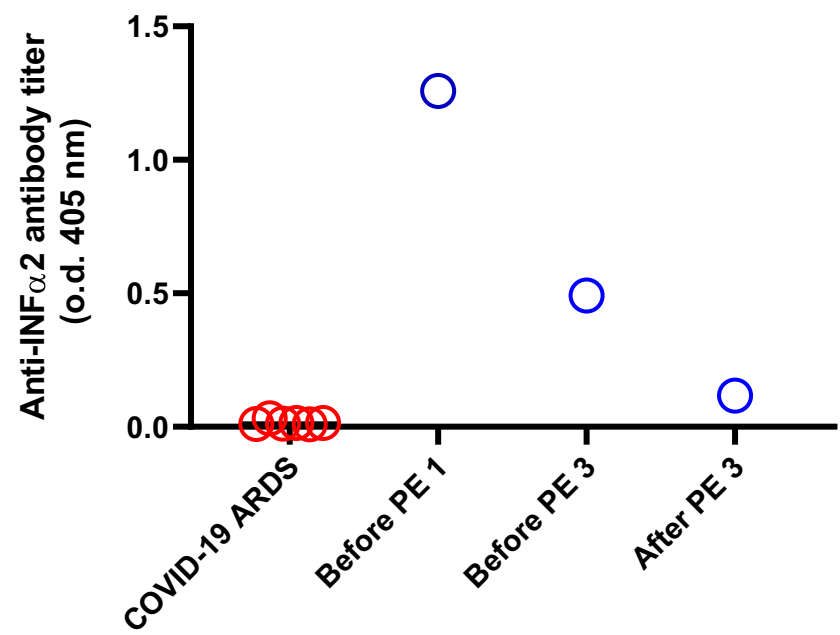

Fig. 3 Concentration of antibodies against type I interferon (IFN) in tracheal aspirates. Measurements were performed in five critically ill patients with COVID-19-associated acute respiratory distress syndrome (COVID-19 ARDS, red circles) and no detectable serum anti-IFN antibodies, and in one patient (patient 4, blue circles) with detectable anti-IFN antibodies, at three time points: before the first and third plasma exchanges (PEs) and after the third plasma exchange. Anti-IFN antibody concentrations were expressed in optical density (o.d.)

patients. One of our patients developed hemorrhagic shock, revealing a spontaneous hemothorax. This rare complication has been reported in COVID-19 patients before [17], but we cannot rule out the possibility that PE may have precipitated this complication. This case highlights the need for further assessments of the benefit-to-risk ratio of this intervention in controlled trials. We found that PE was associated with a decrease in anti-IFN auto-Ab levels, and, to a lesser extent, with a restoration of endogenous serum IFN concentrations. Nevertheless, several concerns remain. First, it remains unknown whether this treatment decreases viral load, which might itself lead to clinical improvement and, possibly, a better prognosis. Indeed, we cannot conclude that PE had a positive clinical impact in our patients. Second, three of our four patients underwent four sessions of PE, but this course of treatment was decided arbitrarily, and a larger number of sessions might have had a clearer beneficial effect. Finally, the precise timing of such treatment, if effective, remains to be determined. Indeed, in addition to removing auto-Abs against type I IFNs, PE in COVID-19 patients could theoretically act on various targets, thereby affecting coagulation [18], inflammatory mediators $[6,7]$, or antiviral innate immunity, as described here. The timing of PE may, therefore, be crucial: for the removal of auto-Abs against type I IFNs and the restoration of type I IFN antiviral immunity, treatment should probably be performed earlier in the course of the disease, when the virus is still in the early phases of replication, viral load is 


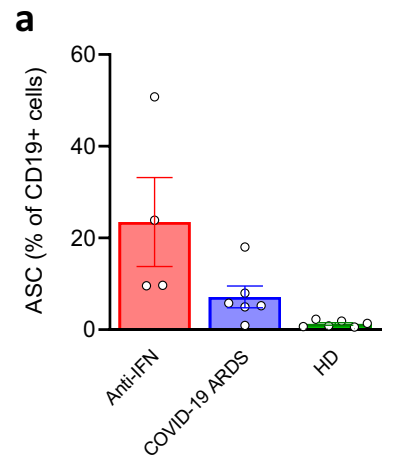

b

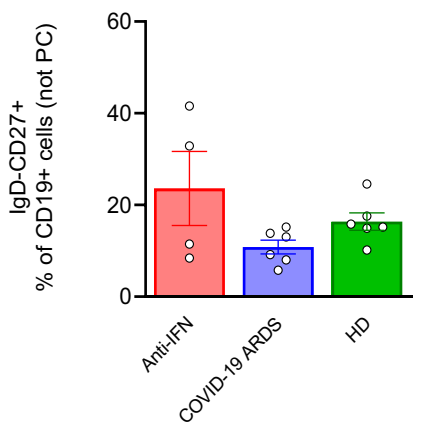

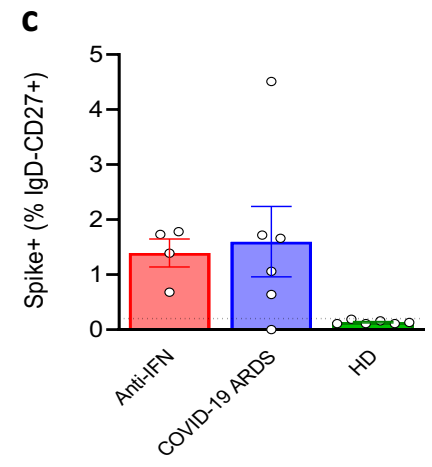

Fig. 4 Patients with life-threatening COVID-19 and type I anti-interferon (IFN) elicit a normal B cell response against SARS-Cov2 spike (S) protein. a Proportion of circulating antibody-secreting cells (ASC). b Proportion of switched IgD-CD27+ memory cells. c Proportion of SARS-CoV-2 S-specific IgD-CD27+ cells among CD19+ cells in four

highest, and the patients have not yet become critically ill. It therefore seems probable that PE was performed too late for maximal benefit in our patients (i.e., PE was initiated between day 8 (patient 4) and day 22 (patient 1) of ICU admission). We thus speculate that in critically ill patients with auto-Abs against type I IFNs, future studies aimed at assessing the effect of PE should initiate the intervention as early as possible. Of note, the time elapsed between the first symptoms of disease and ICU admission was shorter in our patients (from 3 days in patient 2 to 7 days in patient 3 ) than in a general population of critically ill COVID-19 patients (median: 9 [quartile 1: $6-$ quartile 3: 12] in 4244 patients) [19]. Moreover, in the later COVID-ICU cohort, a shorter time between first symptoms to ICU admission was an independent factor associated with day-90 mortality [19].

Our study has a number of limitations. This is an observational cohort study that included a small number of patients $(n=4)$, thus limiting the conclusions that can be drawn. Including control patients with severe SARS-CoV-2 infections and auto-Abs against type I IFNs but not subjected to PE would have allowed for comparing the time course of concentrations of auto-Abs and SARS-CoV-2 replication, and clinical outcomes. However, in the current study the decision to perform PE was made on the bed side and was taken for all four patients with positive auto-Abs given the severity of their clinical condition. It is finally also possible that serum auto-Ab concentrations might have been altered by dexamethasone treatment and ECMO membranes.

\section{Conclusion}

PE efficiently removes auto-Abs against type I IFNs, including those detected in tracheal aspirates, without affecting antiSARS-CoV-2 Ab levels, in patients with life-threatening COVID-19 pneumonia. It was not possible to assess the patients with life-threatening COVID-19 and auto-Abs to type I IFNs (Anti-IFN) compared with six patients with life-threatening COVID-19 (COVID-19 ARDS) and six pre-pandemic healthy donor controls (HD). Each dot represents one patient; bars indicate mean with SEM

clinical impact of this intervention due to the small number of patients studied. Further studies are needed to assess whether the removal of these auto-Abs by PE earlier in the course of infection might be beneficial in patients with auto-Abs against type I IFNs.

Supplementary Information The online version contains supplementary material available at https://doi.org/10.1007/s10875-021-00994-9.

Acknowledgements We thank the patients and their families for placing their trust in us. We thank Y. Nemirovskaya, D. Papandrea, M. Woollett, D. Liu, C. Rivalain, and C. Patissier for administrative assistance. We thank Dr. Dehbia Menouche, Hôpital Henri Mondor, Créteil, for performing the plasma exchanges; Prof. Benjamin Terrier, Hôpital Cochin, Paris, for his participation in the clinical discussions; and Profs. Guillaume Carteaux, Keyvan Razazi, and Nicolas Mongardon, Hôpital Henri Mondor, Créteil, and all the clinicians and nurses involved in the management of the patients.

Author Contribution NDP, PB, RA, JLC, and CEL conceptualized and designed the study, supervised and analyzed the data, wrote the manuscript, and gave approval of the final version to be submitted; PB, SF, $\mathrm{MM}, \mathrm{SB}, \mathrm{KD}$, IA, and IF performed lab experiments, reviewed the manuscript, and gave approval of the final version to be submitted; NDP, RA, and CEL collected the clinical data, reviewed the manuscript, and gave approval of the final version to be submitted; GG, JLC, AC, and AMD reviewed the manuscript and gave approval of the final version to be submitted.

Funding The Laboratory of Human Genetics of Infectious Diseases is supported by the Howard Hughes Medical Institute, The Rockefeller University, the St. Giles Foundation, the National Institutes of Health (NIH) (R01AI088364), the National Center for Advancing Translational Sciences (NCATS), the NIH Clinical and Translational Science Award (CTSA) program (UL1 TR001866), a Fast Grant from Emergent Ventures, the Mercatus Center at George Mason University, the Yale Center for Mendelian Genomics and the GSP Coordinating Center funded by the National Human Genome Research Institute (NHGRI) (UM1HG006504 and U24HG008956), the French National Research Agency (ANR) under the Investments for the Future program (ANR-10-IAHU-01), the Integrative Biology of Emerging Infectious Diseases Laboratory of Excellence (ANR-10-LABX-62- IBEID), the 
French Foundation for Medical Research (FRM) (EQU201903007798), the FRM and ANR GENCOVID project (ANRS-COV05), the Square Foundation, Grandir - Fonds de solidarite pour l'enfance, the SCOR Corporate Foundation for Science, the Institut Institut National de la Santé et de la Recherche Médicale (INSERM), and the University of Paris. P.Ba. was supported by the French Foundation for Medical Research (FRM, EA20170638020) and the M.D.-Ph.D. program of the Imagine Institute (with the support of the Fondation BettencourtSchueller)

Availability of Data and Material All data presented in the current manuscript is available on request to the corresponding author.

Code Availability Not applicable

\section{Declarations}

Conflict of Interest NDP, PB, RA, SF, MM, SB, KD, GG, YTL, IA, IF, and JLC have no conflict of interest to disclose; AC reports grants and personal fees from GETINGE, personal fees from BAXTER, and personal fees from XENIOS, outside the submitted work; CEL reports personal fees from Bayer Healthcare, personal fees from Merck, personal fees from Biomérieux, personal fees from Carmat, personal fees from Thermofischer Brahms, and personal fees from Aerogen, outside the submitted work.

Ethics Approval All subjects were recruited according to protocols approved by the competent local institutional review boards.

Consent to Participate All protocols conformed to local ethics recommendations and informed consent was obtained when required.

Consent for Publication Included subjects were informed and consented for the publication when feasible.

\section{References}

1. Bastard P, Rosen LB, Zhang Q, Michailidis E, Hoffmann H-H, Zhang Y, et al. Autoantibodies against type I IFNs in patients with life-threatening COVID-19. Science. 2020;370:eabd4585.

2. Zhang Q, Bastard P, Liu Z, Le Pen J, Moncada-Velez M, Chen J, et al. Inborn errors of type I IFN immunity in patients with lifethreatening COVID-19. Science. 2020;370:eabd4570.

3. Zhang Q, Bastard P, Bolze A, Jouanguy E, Zhang S-Y. COVID Human Genetic Effort, et al. Life-threatening COVID-19: defective interferons unleash excessive inflammation. Med. 2020;1:14-20.

4. Levy R, Bastard P, Lanternier F, Lecuit M, Zhang S-Y, Casanova JL. IFN-a2a therapy in two patients with inborn errors of TLR3 and IRF3 infected with SARS-CoV-2. J Clin Immunol. 2020; in press.

5. WHO Solidarity Trial Consortium, Pan H, Peto R, Henao-Restrepo A-M, Preziosi M-P, Sathiyamoorthy V, et al. N Engl J Med. 2020; in press.
6. Morath C, Ma W, Zeier M, Speer C, Tiwari-Heckler S, Merle U. Plasma exchange in critically ill COVID-19 patients. Crit Care. 2020;24:481.

7. Gluck WL, Callahan SP, Brevetta RA, Stenbit AE, Smith WM, Martin JC, et al. Efficacy of therapeutic plasma exchange in the treatment of penn class 3 and 4 cytokine release syndrome complicating COVID-19. Respir Med. 2020;175:106188.

8. Khamis F, Al-Zakwani I, Al Hashmi S, Al Dowaiki S, Al Bahrani M, Pandak N, et al. Therapeutic plasma exchange in adults with severe COVID-19 infection. Int J Infect Dis. 2020;99:214-8.

9. Keith PD, Scott LK, Weaver KE, Day M, Choe C, Perkins L, et al. Treatment of critically ill coronavirus disease 2019 patients with adjunct therapeutic plasma exchange: a single-center retrospective case series. Crit Care Explor. 2020;2:e223.

10. Stahl K, Bode C, David S. First do no harm-beware the risk of therapeutic plasma exchange in severe COVID-19. Crit Care. 2020;24:363.

11. Lescure F-X, Bouadma L, Nguyen D, Parisey M, Wicky P-H, Behillil S, et al. Clinical and virological data of the first cases of COVID-19 in Europe: a case series. Lancet Infect Dis. 2020;20: $697-706$.

12. RECOVERY Collaborative Group, Horby P, Lim WS, Emberson JR, Mafham M, Bell JL, et al. Dexamethasone in Hospitalized Patients with Covid-19 - Preliminary Report. N Engl J Med. 2020; in press.

13. Corman VM, Landt O, Kaiser M, Molenkamp R, Meijer A, Chu DK, et al. Detection of 2019 novel coronavirus (2019-nCoV) by real-time RT-PCR. Euro Surveill. 2020;25.

14. Rissin DM, Kan CW, Campbell TG, Howes SC, Fournier DR, Song L, et al. Single-molecule enzyme-linked immunosorbent assay detects serum proteins at subfemtomolar concentrations. Nat Biotechnol. 2010;28:595-9.

15. Mathian A, Mouries-Martin S, Dorgham K, Devilliers H, Barnabei $\mathrm{L}$, Ben Salah E, et al. Monitoring disease activity in systemic lupus erythematosus with single-molecule array digital enzyme-linked immunosorbent assay quantification of serum interferon- $\alpha$. Arthritis Rheumatol. 2019;71:756-65.

16. Fourati S, Hue S, Pawlotsky J-M, Mekontso-Dessap A, de Prost N. SARS-CoV-2 viral loads and serum IgA/IgG immune responses in critically ill COVID-19 patients. Intensive Care Med. 2020;46: 1781-3.

17. Desnos C, Boussouar S, Hekimian G, Redheuil A, Combes A Spontaneous hemothorax in 4 COVID-19 ARDS patients on VVECMO revealing pulmonary artery aneurysms. Crit Care. 2020;24: 638.

18. Bowles L, Platton S, Yartey N, Dave M, Lee K, Hart DP, et al. Lupus anticoagulant and abnormal coagulation tests in patients with Covid-19. N Engl J Med. 2020;383:288-90.

19. COVID-ICU Group on behalf of the REVA Network and the COVID-ICU Investigators. Clinical characteristics and day- 90 outcomes of 4244 critically ill adults with COVID-19: a prospective cohort study. Intensive Care Med. 2020;47:60-73.

Publisher's Note Springer Nature remains neutral with regard to jurisdictional claims in published maps and institutional affiliations. 